\title{
A new computational method based on the minimum lithostatic deviation (MLD) principle to analyse slope stability in the frame of the 2-D limit-equilibrium theory
}

\author{
S. Tinti and A. Manucci \\ Università degli Studi di Bologna, Dipartimento di Fisica, Settore di Geofisica, Viale Berti Pichat, 8 - 40127 Bologna, Italy
}

Received: 18 January 2008 - Revised: 2 June 2008 - Accepted: 4 June 2008 - Published: 16 July 2008

\begin{abstract}
The stability of a slope is studied by applying the principle of the minimum lithostatic deviation (MLD) to the limit-equilibrium method, that was introduced in a previous paper (Tinti and Manucci, 2006; hereafter quoted as TM2006). The principle states that the factor of safety $F$ of a slope is the value that minimises the lithostatic deviation, that is defined as the ratio of the average inter-slice force to the average weight of the slice. In this paper we continue the work of TM2006 and propose a new computational method to solve the problem. The basic equations of equilibrium for a 2-D vertical cross section of the mass are deduced and then discretised, which results in cutting the cross section into vertical slices. The unknowns of the problem are functions (or vectors in the discrete system) associated with the internal forces acting on the slice, namely the horizontal force $E$ and the vertical force $X$, with the internal torque $A$ and with the pressure on the bottom surface of the slide $P$. All traditional limit-equilibrium methods make very constraining assumptions on the shape of $X$ with the goal to find only one solution. In the light of the MLD, the strategy is wrong since it can be said that they find only one point in the searching space, which could provide a bad approximation to the MLD. The computational method we propose in the paper transforms the problem into a set of linear algebraic equations, that are in the form of a block matrix acting on a block vector, a form that is quite suitable to introduce constraints on the shape of $X$, but also alternatively on the shape of $E$ or on the shape of $A$. We test the new formulation by applying it to the same cases treated in TM2006 where $X$ was expanded in a three-term sine series. Further, we make different assumptions by taking a three-term cosine expansion corrected by the local weight for $X$, or for $E$ or for $A$, and find the corresponding MLDs. In the illustrative applications
\end{abstract}

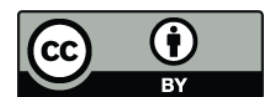

Correspondence to: $\mathrm{S}$. Tinti (stefano.tinti@unibo.it) given in this paper, we find that the safety factors associated with the MLD resulting from our computations may differ by some percent from the ones computed with the traditional limit-equilibrium methods.

\section{Introduction}

Determining the stability of a slope is a problem of great interest since a long time to assess the hazard and mitigate the risk of an area. Different methods of analysis have been developed, ranging from approximated 1-D or 2-D approaches to fully 3-D methods solving visco-plastic equations through finite-element or finite-difference techniques. Usually more sophisticated methods such as 3-D need a very detailed knowledge of the soil and subsoil conditions that are difficult and expensive to acquire, which makes the recourse to simplified techniques often more convenient and more practical to use. The classical limit-equilibrium methods belong to the category of approximated methods. The methods were firstly introduced with the Fellenius formula (1927) and lately developed by Bishop (1955), by Morgenstern and Price (1965), by Spencer (1967), by Janbu (1968) and others. They were the starting point of the slope stability analysis and were successfully applied and repeatedly refined, since they are still subject of active research (e.g. Duncan and Wright, 1980; Chen and Morgenstern, 1983; Leschchinsky and Huang, 1992; Chen et al., 2001; Zhu et al., 2003; Jiang and Yamagami, 2004; Karaulov, 2005; Tinti and Manucci, 2006, hereafter quoted as TM2006; Zheng et al. 2007; Pink, 2007).

Typically limit-equilibrium methods consider vertical sections of the sliding body that are cut into vertical slices. Stability is expressed by the safety factor $F$, that is computed by imposing the equilibrium of each slice (though this is known to be only a necessary, but not a sufficient condition). A theoretical problem for this approach is that in the set formed

Published by Copernicus Publications on behalf of the European Geosciences Union. 


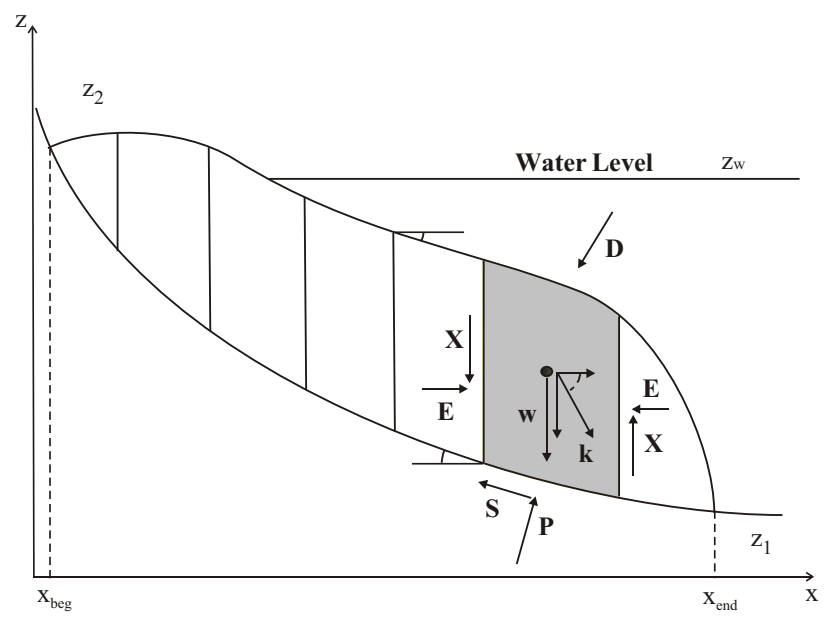

Fig. 1. Sketch of the vertical cross-section of the sliding body cut into slices. Cartesian coordinates $x$ and $z$ are taken to increase rightward and upward, as usual. The forces, described in the text, act on each slice. In the discretised version of the analytical problem, slices are defined as the portions of the body comprised between the vertical planes $x_{i}-\Delta x / 2$ and $x_{i}+\Delta x / 2$ The seismic load is a force proportional to the weight of the slice and forms the angle $\psi$ with the horizontal. The load $D$ is a pressure acting on the body top surface.

by all balance equations the number of unknowns (i.e. the safety factor and the component of the forces and torques acting on the slices) is greater than the number of equations with the consequence that the solution, and therefore even the computed safety factor, is not unique. This is usually overcome by means of ad-hoc assumptions (constraints), that differ from one author to the other, and the correspondent resulting safety factors may depart by $5-10 \%$ from one another. In a previous paper (TM2006) we have called the attention on this issue and we have proposed a drastic change of view for the limit-equilibrium approach. The factor of safety, instead of an unknown, was proposed to be treated as a known parameter, say $F^{*}$, that is left to range within a given interval. For each value of $F^{*}$, a solution, say $S\left(F^{*}\right)$, to the equilibrium equations is computed. The unique solution to the problem is found by introducing and applying a new criterion, that was called the "Principle of Minimum Lithostatic Deviation (MLD)". The lithostatic deviation $\delta$ expresses the ratio of the average magnitude of the internal slice-slice forces and the total weight of the sliding mass (see formula (18) of TM2006). The MLD principle means that one calculates the value of $\delta$ for each computed solution $S\left(F^{*}\right)$, obtaining the relation $\delta\left(S\left(F^{*}\right)\right.$ ) or more simply $\delta\left(F^{*}\right)$, and, eventually one selects the safety factor $\mathrm{F}$ corresponding to the minimum value of $\delta$, i.e. $F=F^{*}\left(\delta_{\min }\right)$. In the present paper the validity of the MLD criterion is confirmed, but the computational technique used to solve the set of the equilibrium equations, i.e. to obtain the solution $S\left(F^{*}\right)$, is revisited and a procedure based on matrix calculus is introduced that is faster and eas- ier to manipulate than the method previously used and may be easily extended to handle limit-equilibrium analyses for bodies sliding on surfaces (3-D) rather than along profiles (2-D).

\section{Formulation of the problem}

The stability of a body in 2-D is studied by taking into account vertical cross-sections. The section belongs to the plane $(x, z)$ and both, the body and the slope, are assumed to be uniform along the horizontal axis $y$. In the sketch of Fig. 1 a vertical section is given where the body is confined between the sliding surface $z_{1}(x)$ and the upper surface $z_{2}(x)$. In limit-equilibrium analysis the section is subdivided into vertical slices and the governing system of equations is obtained by imposing the balance of all forces and torques acting on these slices.

\subsection{The set of equilibrium equations}

We use the same formalism we adopted in TM2006 where the reader may find a detailed derivation of the basic set of equations. Here we limit to recall the notions essential to understand the subsequent analysis. Forces are depicted in Fig. 1: $w(x)$ is the slice weight per unit area, $D(x)$ is an external pressure acting on the upper slide surface, $P(x)$ and $S(x)$ are respectively the pressure and the shear stress at the base of the slice, $E(x)$ and $X(x)$ are the horizontal and vertical components of the inter-slice forces, while $A(x)$ is the component of the torque along the axis $y$. They are functions of the horizontal coordinate $x$, and each slice is taken to have an infinitesimal width $\Delta x$, a finite height $z_{2}(x)-z_{1}(x)$ and to be comprised between $x-1 / 2 \Delta x$ and $x+1 / 2 \Delta x$.

The set of equilibrium equations may be given the following expression (see TM2006):

$$
\begin{aligned}
& \frac{d}{d x} E+P \tan \alpha-S-D \tan \beta=-w k \cos \psi \\
& \frac{d}{d x} X+P+S \tan \alpha-D=(1+k \sin \psi) w \\
& \frac{d}{d x} A-z_{1} \frac{d}{d x} E-X-D \tan \beta\left(z_{2}-z_{1}\right)=-w k \cos \psi\left(z_{B}-z_{1}\right) \\
& F S=\bar{c}^{*}+P \tan \bar{\phi}^{\prime}
\end{aligned}
$$

with

$\bar{c}^{*}=\bar{c}^{\prime}-u \tan \bar{\phi}^{\prime}$

Eqations (1) and (2) result from the balance of the horizontal and vertical components of the forces, whereas Eq. (3) expresses the balance of the torque. The last Eq. (4) is the essence of the limit-equilibrium theory. This assumes that, when body conditions are close to instability, the shear stress $S$ and the shear strength $S_{\max }$ tend to be equal in all points of 
the basal surface. According to the Mohr-Coulomb law, $S_{\max }$ is given by:

$S_{\max }(x)=\bar{c}^{\prime}(x)+(P(x)-u(x)) \tan \bar{\phi}^{\prime}(x)$

where $u(x)$ is the pore pressure dependent on the piezometric level, and $\bar{c}^{\prime}$ and $\bar{\phi}^{\prime}$ are average values of the material cohesion and of the friction angle, as better explained in Sect. 2.2. Consistently with this view, Eq. (4) imposes that shear strength and stress are proportional via the coefficient $F$, the safety factor, and that $F$ may be used to mark the boundary between stable $(F>1)$ and unstabe $(F<1)$ regions.

In Eqs. (1)-(3) $\alpha(x)$ and $\beta(x)$ are the angle of slope respectively at the base and at the top of the slice. The slice weight $w$ is defined as:

$w(x)=g \int_{z_{1}}^{z_{2}} \rho(x, z) d z$

where $\rho$ is the density that depends on the position $(x, z)$ in heterogeneous bodies.

The torque $A(x)$ is computed with respect to the centre of mass of the slide $z_{B}(x)$ which is given by:

$z_{B}(x)=\frac{\int_{z_{1}}^{z_{2}} \rho(x, z) z d z}{w(x)}$

The load $D(x)$ on the upper surface, when it is due to water as in the case of a body that is totally or partially submerged, can be expressed as follows:

$$
\begin{aligned}
& D(x)=\rho_{w} g\left[z_{w}-z_{2}(x)\right] \quad z_{2}(x)<z_{w} \\
& D(x)=0 \quad z_{2}(x) \geq z_{w}
\end{aligned}
$$

where $z_{w}$ is the water level and $\rho_{w}$ the water density.

Equations (1)-(3) account also for the seismic load, which is assumed to act at the centre of mass of the slice along the direction $\psi$ and to be proportional to the slice weight through the coefficient $k$.

The set of Eqs. (1)-(4) is the basic system for the slope stability problem according to the limit-equilibrium method. It has to be complemented by the boundary conditions, stating that all the inter-slice forces and torques vanish at the beginning and at the end of the sliding body:

$$
\begin{aligned}
& E\left(x_{\text {beg }}\right)=E\left(x_{\text {end }}\right)=0 \\
& X\left(x_{\text {beg }}\right)=X\left(x_{\text {end }}\right)=0 \\
& A\left(x_{\text {beg }}\right)=A\left(x_{\text {end }}\right)=0
\end{aligned}
$$

Equations (1)-(7) form a set of three first-order ordinary differential equations completed by the corresponding boundary conditions and by an additional relationship, Eq. (4). This set contemplates one unknown parameter, $F$, and five unknown functions defined in the finite domain $\left[x_{\mathrm{beg}}, x_{\mathrm{end}}\right]$, namely the basal stresses $P(x)$ and $S(x)$ and forces and torques associated with slice interaction $E(x), X(x)$ and $A(x)$. In this formulation, the sliding surface described by the function $z_{1}(x)$ is considered to be known a priori: it may be circular, as it is assumed in some classical limitequilibrium methods, or have a more general shape (see Graham, 1984).

\subsection{The stratified soil}

Assuming a homogeneous sliding body is often an oversimplification of the problem and may lead to questionable conclusions on slope stability, especially if there is evidence of the existence of weak layers at some depth. Accounting for a stratified soil in the formulation given in Sect. 2.1 is straightforward. Let us suppose that the body cross-section is composed of $M$ layers with layer $i$ lying between interfaces $i$ and $i+1$, and with interfaces described by the functions $z_{\text {int }, i}(x)$ and $z_{\text {int }, i+1}(x)\left(z_{\text {int }, i}<z_{\text {int }, i+1}\right)$. We can further suppose that the body base $z_{1}$ and top $z_{2}$ coincide with surfaces $z_{\text {int, } 1}(x)$ and $z_{\text {int, } M+1}(x)$. Material properties such as density $\rho$, cohesion $c^{\prime}$ and friction angle $\phi^{\prime}$ will change from one layer to the next, but incorporating the depth-dependence in the stability equations is quite easy. The expression for the weight $w(x)$ in the previous section is still valid and the integral will reduce to a summation across all layers of the material column $x$ (that is formed at most by $M$ layers). As for cohesion and friction angle, it is remarked that only the values they assume on the sliding surface $z_{1}(x)$ are of relevance in Eq. (4), i.e. the values $c^{\prime}\left(x, z_{1}(x)\right) \phi^{\prime}\left(x, z_{1}(x)\right)$. Practically, since the set of Eqs. (1)-(4) cannot be solved analytically, but through numerical methods, it is anticipated here that covering the computational domain $\left[x_{\text {beg }}, x_{\text {end }}\right]$ with a finite grid with $\mathrm{N}+1$ nodes, implies the partition of the body cross-section into $\mathrm{N}$ vertical slices. Such a discretization implies further that, given the slice corresponding to the interval $\left[x_{i}, x_{i+1}\right]$, in place of local values of cohesion and of friction angle, average values $\bar{c}^{\prime}$ and $\bar{\phi}^{\prime}$ are to be used, with average computed over the base of the slide, according to the formulas:

$\bar{c}_{i}^{\prime}=\frac{\int_{x_{i}}^{x_{i+1}} c^{\prime}\left(x, z_{1}(x)\right) d x}{x_{i+1}-x_{i}}$

$\bar{\phi}_{i}^{\prime}=\frac{\int_{x_{i}}^{x_{i+1}} \phi^{\prime}\left(x, z_{1}(x)\right) d x}{x_{i+1}-x_{i}}$

For a stratified body, these integrals will reduce to a sum extended to all layers intersecting the base of the $i$-th slice of the numerical partition. 


\subsection{The traditional methods}

Traditional methods of limit equilibrium treat the safety factor as an unknown parameter. The solution to the set of Eqs. (1)-(7) is underdetermined since there are four equations for five unknown functions of $x$ and for one unknown parameter, $F$. Despite the existence of more than one solution, the first methods were devised in times when numerical computing was still a very hard job and introduced drastic simplification to allow the computation of at least one solution. Fellenius (1927) assumed that inter-slice forces are null; Bishop (1955) and Janbu (1968) posed vertical forces $X$ equal to zero and disregarded the horizontal and the vertical equilibrium equations, respectively. On the other hand, Morgenstern and Price (1965) and Spencer (1967) were the first who tackled the non-uniqueness problem and overcame it by imposing a relationship between the vertical and horizontal components of the inter-slice forces, which is equivalent to add a new equation to the original set (1)-(7). Spencer's method is taken in this article as representative of classical methods and against it we will compare our results.

\section{Determination of the unique solution}

The non-uniqueness of the solution has the consequence that also the safety factor $F$, that is one of the unknowns of the problem, cannot be determined univocally. It can be shown that usually one can find exact solutions to the Eqs. (1)(7) with very different values of $F$, ranging from below to above the critical value of 1 . And this in principle is a relevant theoretical weakness of this approach, undermining the meaning itself of the analysis. In practice, the additional hypotheses introduced by Morgenstern and Price, by Spencer and by others, restrict the interval of variability of the safety factor to more acceptable limits. In TM2006 a totally different approach was suggested, converting the limit-equilibrium method to a minimization problem, where $F$ is treated as a free parameter, and not as an unknown. Within a given interval of $F$, say $\left[F_{\min }, F_{\max }\right]$, one searches the solution to the set of Eqs. (1)-(7) that minimizes the lithostatic deviation $\delta$, and the value of the parameter $F$ corresponding to the minimum value of $\delta$ is taken as the final result of the limit-equilibrium analysis. The lithostatic deviation is defined as:

$\delta=W^{-1}\left[\frac{1}{\left(x_{\text {end }}-x_{\text {beg }}\right)} \int_{x_{\text {beg }}}^{x_{\text {end }}}\left(E(x)^{2}+X(x)^{2}\right) d x\right]^{1 / 2}$

where:

$$
W=\frac{1}{x_{\text {end }}-x_{\text {beg }}} \int_{x_{\text {beg }}}^{x_{\text {end }}} w(x) d x
$$

Notice that $\delta$ is the dimensionless ratio of the average magnitude of the internal forces to the total weight $W$ of the slid- ing mass. Notice further that the condition $\delta=0$ trivially implies that both $E(x)$ and $X(x)$ are identically zero over the domain $\left[x_{\text {beg }}, x_{\text {end }}\right]$ which is a state of equilibrium only for the special case of a body of uniform thickness lying over a uniform slope.

\section{Finding the solution}

\subsection{The discretization}

The system of Eqs. (1)-(7) can be set in a more adequate form. In first place, we note that Eq. (4) involves the known parameter $F$, the known material properties $\left(\bar{c}^{\prime}, \bar{\phi}^{\prime}\right.$ and $\left.u\right)$ and two unknown functions $P(x)$ and $S(x)$. Hence, with the aid of Eq. (4) one can express $S(x)$ in terms of $P(x)$, and replace it in all Eqs. (1)-(3). After some manipulations one obtains the following system of equations in the unknown $E(x), X(x), A(x)$ and $P(x)$ :

$\frac{d E}{d x}+P \alpha_{E}=\beta_{E}$

$\frac{d X}{d x}+P \alpha_{X}=\beta_{X}$

$\frac{d A}{d x}+P \alpha_{A}-X=\beta_{A}$

including coefficients that are given by:

$\alpha_{E}=\tan \alpha-\frac{\tan \bar{\phi}^{\prime}}{F}$

$\beta_{E}=\frac{\bar{c}^{*}}{F}+D \tan \beta-k \cos \psi w$

$\alpha_{X}=1+\frac{\tan \alpha \tan \bar{\phi}^{\prime}}{F}$

$\beta_{X}=D-\frac{\bar{c}^{*}}{F} \tan \alpha+(1+k \sin \psi) w$

$\alpha_{A}=z_{1} \alpha_{E}$

$\beta_{A}=D \tan \beta\left(z_{2}-z_{1}\right)-k \cos \psi\left(z_{B}-z_{1}\right) w+z_{1} \beta_{E}$

Observe that all such coefficients are known functions of the problem, since they depend on the geometry of the body, on its material properties, on the external loads (water layer and seismic forcing) and on the parameter $F$. Observe further that some coefficients, such as $\alpha_{E}$ and $\alpha_{X}$, are dimensionless, while others are not: $\alpha_{A}$ is a length, $\beta_{E}$ and $\beta_{X}$ are pressures, while $\beta_{A}$ is a pressure times a length.

The solution is searched for by numerical means. The computational domain $\left[x_{\text {beg }}, x_{\text {end }}\right]$ is discretised into $N$ equal intervals of length $\Delta x$ through the nodal points $x_{i}, i \in$ $[0, N]$, which entails that the body is cut into $N$ vertical slices, and that all variables result to be accordingly discretised. Note that $x_{0}=x_{\text {beg }}$ and that $x_{N}=x_{\text {end }}$.In such discretization process, it is convenient to take the pressure $P$ and all the coefficients given by the relationships (9) at the mid-points of the intervals, while the inter-slice forces $E(x)$, $X(x)$ and the torque $A(x)$ are taken at the nodal points. As a consequence, we may introduce the $\mathrm{N}$-component vector 
Table 1. Comparison of stability analysis results obtained with the traditional Spencer method, the Tinti and Manucci (T\&M) method and with the various applications of the new computational method presented in this paper. The slope is the same as in TM2006. Case 1: no seismic and no water load. Case 2: water load only. Case 3: seismic load only. The best results (i.e. the MLDs) are found in the cells with bold characters. All are in the same raw, since are obtained with the same method.

\begin{tabular}{lllllll}
\hline \multirow{2}{*}{ Method } & \multicolumn{2}{c}{ Case 1} & \multicolumn{2}{c}{ Case2 } & \multicolumn{2}{c}{ Case 3 } \\
& F & $\delta$ & F & $\delta$ & F & $\delta$ \\
\hline Spencer & 1.468 & 0.1052 & 1.579 & 0.1163 & 0.984 & 0.1780 \\
T\&M & 1.409 & 0.0772 & 1.510 & 0.0845 & 0.925 & 0.1193 \\
T\&M-new & 1.409 & 0.0776 & 1.509 & 0.0847 & 0.926 & 0.1199 \\
T\&M-Xsin & 1.409 & 0.0776 & 1.509 & 0.0847 & 0.926 & 0.1199 \\
T\&M-Xcos & $\mathbf{1 . 4 0 5}$ & $\mathbf{0 . 0 7 6 2}$ & $\mathbf{1 . 5 0 5}$ & $\mathbf{0 . 0 8 3 5}$ & $\mathbf{0 . 9 2 0}$ & $\mathbf{0 . 1 1 4 7}$ \\
T\&M-Ecos & 1.415 & 0.0836 & 1.510 & 0.0891 & 0.922 & 0.1305 \\
T\&M-Acos & 1.412 & 0.0812 & 1.522 & 0.0885 & 0.937 & 0.1301 \\
\hline
\end{tabular}

$\boldsymbol{p}$, with $p_{i}=p\left(x_{i}-\Delta x / 2\right), i \in[1, N]$, and in an analogous way the vector $\boldsymbol{b}_{\boldsymbol{E}}$ with $b_{E, i}=\beta_{E}\left(x_{i}-\Delta x / 2\right)$, the vector $\boldsymbol{b}_{X}$ with $b_{X, i}=\beta_{X}\left(x_{i}-\Delta x / 2\right)$ and the vector $\boldsymbol{b}_{\boldsymbol{A}}$ with $b_{A, i}=\beta_{A}\left(x_{i}-\Delta x / 2\right)$. Similar discretization holds for the coefficient $\alpha_{E}$, but, as will be seen later, instead of a vector it is more adequate to introduce a diagonal $N \times N$ matrix $\mathbf{A}_{\mathbf{E}}$ with $\left(A_{E}\right)_{i, i}=\alpha_{E}\left(x_{i}-\Delta x / 2\right)$. Analogously we define the matrices $\mathbf{A}_{\mathbf{X}}$ and $\mathbf{A}_{\mathbf{A}}$. We may also introduce $(N+1)$-component vectors for forces and torque, but, since these are null at the boundaries in force of conditions (5)-(7), we are allowed to restrict the attention to the internal nodes of the domain. Hence, the unknown function $E(x)$ is transformed into the (N-1)-component vector $\boldsymbol{e}$ with $e_{i}=E\left(x_{i}\right), i \in[1, N-1]$, and the same applies to vectors $\boldsymbol{X}$ and $\boldsymbol{a}$, i.e. $X_{i}=X\left(x_{i}\right)$ and $a_{i}=A\left(x_{i}\right)$. In terms of these discretized quantities, it is easy to transform the first of the differential Eq. (8), namely the one concerning the equilibrium of the slices along the horizontal axis, into the following system of algebraic equations:

$$
\left\{\begin{array}{l}
e_{1}+p_{1} \alpha_{E, 1} \Delta x=\beta_{E, 1} \Delta x \\
\cdots . \\
e_{i}-e_{i-1}+p_{i} \alpha_{E, i} \Delta x=\beta_{E, i} \Delta x \\
\cdots \\
e_{N-1}-e_{N-2}+P_{N-1} \alpha_{E, N-1} \Delta x=\beta_{E, N-1} \Delta x \\
-e_{N-1}+p_{N} \alpha_{E, N} \Delta x=\beta_{E, N} \Delta x
\end{array}\right.
$$

After introducing the rectangular $N \times(N-1)$ matrix $\Gamma$ given by:

$$
\boldsymbol{\Gamma}=\left(\begin{array}{ccccc}
1 & 0 & 0 & 0 & 0 \\
-1 & 1 & 0 & 0 & 0 \\
0 & -1 & 1 & 0 & 0 \\
\ldots & \ldots & \cdots & \cdots & \cdots \\
0 & 0 & 0 & -1 & 1 \\
0 & 0 & 0 & 0 & -1
\end{array}\right)
$$

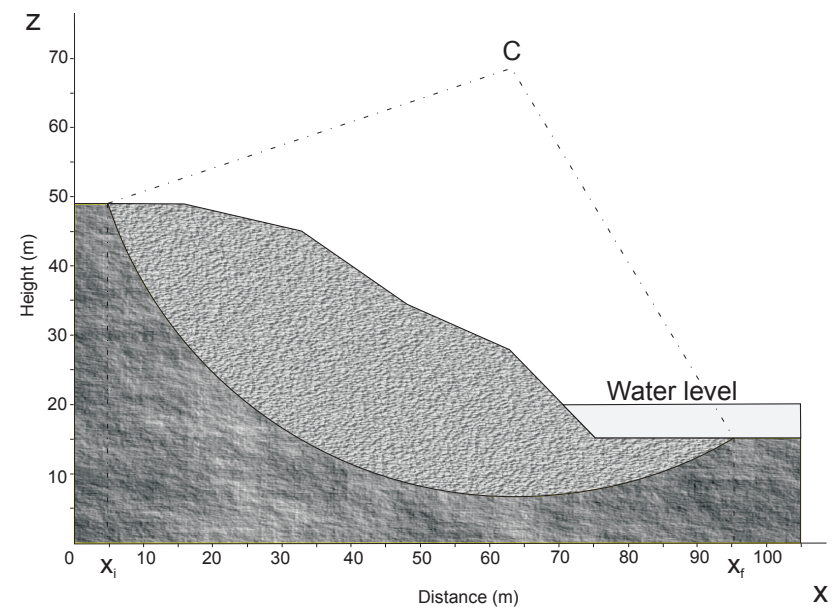

Fig. 2. Vertical cross-section of the body already analysed in TM2006. The sliding mass is in light grey. The sliding surface has a circular profile centred in C. The soil parameters $c^{\prime}, \phi^{\prime}$ and $\rho$ are constant, with respective values of $6 \mathrm{kPa}, 25^{\circ}$ and $25 \mathrm{kNm}^{-3}$. The sliding body is partially submerged by a $5 \mathrm{~m}$ deep water layer.

the system (10) can be written in the following vectorial form:

$\boldsymbol{\Gamma e}+\mathbf{A}_{\mathbf{E}} \mathbf{p} \Delta x=\mathbf{b}_{\mathbf{E}} \Delta x$

Analogously, on discretizing the vertical equilibrium Eq. (2) and accounting for the corresponding boundary condition (6), we obtain:

$\boldsymbol{\Gamma} \mathbf{x}+\mathbf{A} \mathbf{x p} \Delta x=\mathbf{b}_{\mathbf{X}} \Delta x$

In the torque equilibrium Eq. (3), the term $X$ has to be evaluated at the interval mid-points, which is obtained by taking the average value between two adjacent nodes. Bearing this in mind and the boundary condition (7), the following $N-1$ relations can be obtained:

$\Gamma \mathbf{a}+\mathbf{A}_{\mathbf{A}} \mathbf{p} \Delta x-\mathbf{\Omega} \mathbf{X} \Delta x=\mathbf{b}_{\mathbf{A}} \Delta x$

where use is made of the rectangular $N \times(N-1)$ matrix $\boldsymbol{\Omega}$ defined as:

$\boldsymbol{\Omega}=\frac{1}{2}\left(\begin{array}{ccccc}1 & 0 & 0 & 0 & 0 \\ 1 & 1 & 0 & 0 & 0 \\ 0 & 1 & 1 & 0 & 0 \\ \ldots & \cdots & \cdots & \cdots & \cdots \\ 0 & 0 & 0 & 1 & 1 \\ 0 & 0 & 0 & 0 & 1\end{array}\right)$

Putting together the Eqs. (11), (12) and (13), one obtains a set of $3 N$ linear algebraic equations linking as many as $4 N-3$ unknowns, namely $N$ unknown values for $\boldsymbol{p}$ and a total of $3(N-1)$ unknown values for $\mathbf{e}, \mathbf{X}$, and $\mathbf{a}$. The consequence is that there are $N-3$ unknowns more than equations and that, as expected, the discretised version of the problem reflects the underdetermination of the original formulation. 

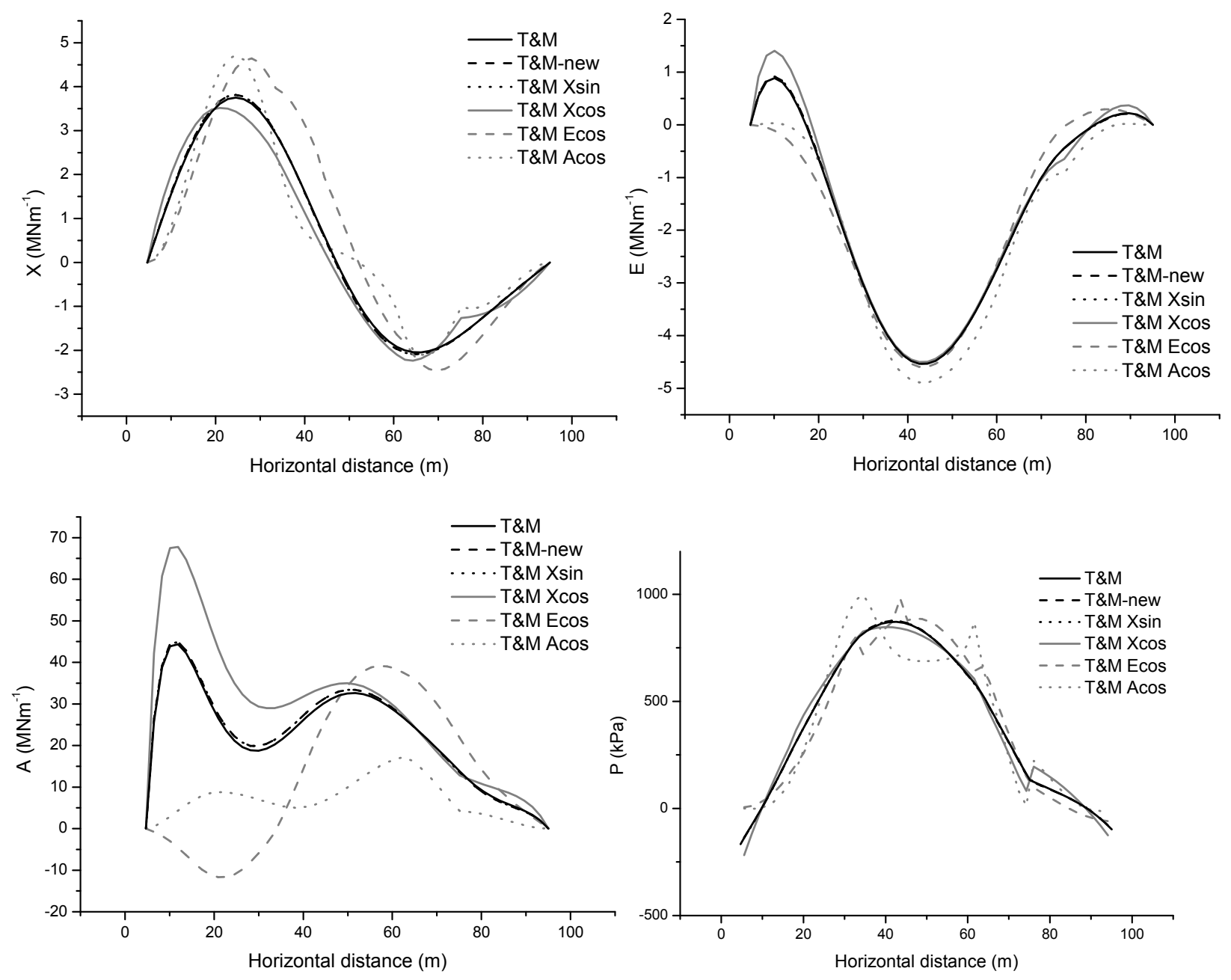

Fig. 3. Graphs of the inter-slice forces $E$ and $X$, of the torque $A$ and of the bottom pressure $P$ corresponding to the best solutions of the various methods applied for Case 1.

\subsection{Non uniqueness of the solution}

In order to reduce the degree of freedom of the algebraic system (11)-(13), some assumptions must be made on the unknowns. In this paper we will consider restricting hypotheses on the form of the inter-slice forces $E$ and $X$, and of the torque $A$. Consistently with classical limit-equilibrium methods, our first assumption regards the vertical forces $X$. Let us expand the function $X(x)$ over a basis of analytical known functions $f_{k}(x)$ (for example, a Fourier series expansion), that is truncated to the first $m$ terms. Correspondingly, the discretised version of such expansion yields the following set of $N$-1 equations:

$X_{1}=\sum_{k=1}^{m} v_{k} f_{k}\left(x_{1}\right)$

$X_{2}=\sum_{k=1}^{m} v_{k} f_{k}\left(x_{2}\right)$
$X_{N-1}=\sum_{k=1}^{m} v_{k} f_{k}\left(x_{N-1}\right)$

this can be seen as a mapping of the $N-1$ unknowns $X$ into the $m$ unknowns $v$, i.e. the coefficients of the truncated expansion. Formally, after defining $f_{i k}=f_{k}\left(x_{i}\right)$, the above relations can be synthesised as:

$X_{i}=\sum_{k=1}^{m} f_{i k} v_{k}$

The position (14), or (15) equivalently, increases the number of equations to $3 N+(N-1)=4 N-1$ and the number of unknowns to $4 N-3+m$, which means that the balance between unknowns and equations is obtained when $m=2$. The interpretation is simple: either we consider a series of only two terms, or we consider a higher order expansion, but only two arbitrary terms of the expansion can be considered unknown coefficients, while the others have to be treated as 


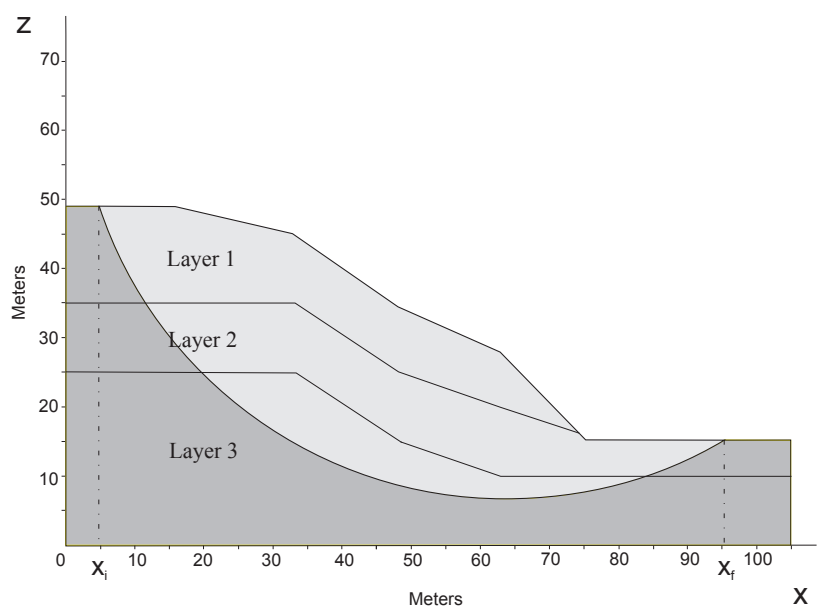

Fig. 4. Vertical cross-section of the three-layer sliding mass. The soil parameters are given in the text and in caption of Table 2.

free parameters. We can therefore make this distinction explicit by writing:

$X_{i}=f_{i 1} v_{1}+f_{i 2} v_{2}+\sum_{k=1}^{m-2} g_{i k} q_{k}$

where the two unknowns are $v_{1}$ and $v_{2}$, and the known parameters and related functions are denoted respectively $q_{k}$ and $g_{i k}$ for sake of clarity.

After introducing the 2-component vector $v$ and the ( $m-2)$ component vector $\boldsymbol{q}$ defined by:

$\boldsymbol{v}=\left(\begin{array}{c}v_{1} \\ v_{2}\end{array}\right) \quad \boldsymbol{q}=\left(\begin{array}{c}q_{1} \\ \cdots \\ q_{m-2}\end{array}\right)$

as well as the $(N-1) \times 2$ matrix $\mathbf{F}_{\mathbf{1}}$ and the $(N-1) \times(m-2) \mathbf{F}_{\mathbf{2}}$ defined as:

$\mathbf{F}_{\mathbf{1}}=\left(\begin{array}{cc}f_{1,1} & f_{1,2} \\ f_{2,1} & f_{2,2} \\ f_{N-1,1} & f_{N-1,2}\end{array}\right)$

$\mathbf{F}_{\mathbf{2}}=\left(\begin{array}{cccc}g_{1,1} & g_{1,2} & \ldots & g_{1, m-2} \\ g_{2,1} & g_{2,2} & \ldots & g_{2, m-2} \\ g_{N-1,1} & g_{N-1,2} & \ldots & g_{N-1, m-2}\end{array}\right)$

Equations (16) can be written in this simple vectorial form:

$\mathbf{X}-\mathbf{F}_{1} \mathbf{v}=\mathbf{F}_{2} \mathbf{q}$

\subsection{Solving the problem}

The final algebraic system of equations can be assembled by putting together the above Eqs. (11)-(13) and (17), which leads to:

$$
\left\{\begin{array}{l}
\Gamma \mathbf{e}+\mathbf{A}_{\mathbf{E}} \mathbf{p} \Delta x=\mathbf{b}_{\mathbf{E}} \Delta x \\
\Gamma \mathbf{X}+\mathbf{A}_{\mathbf{X}} \mathbf{p} \Delta x=\mathbf{b}_{\mathbf{X}} \Delta x \\
\Gamma \mathbf{a}+\mathbf{A}_{\mathbf{A}} \mathbf{p} \Delta x-\mathbf{\Omega} \mathbf{X} \Delta \mathbf{x}=\mathbf{b}_{\mathbf{A}} \Delta x \\
\mathbf{X}-\mathbf{F}_{\mathbf{1}} \mathbf{v}=\mathbf{F}_{\mathbf{2}} \mathbf{q}
\end{array}\right.
$$

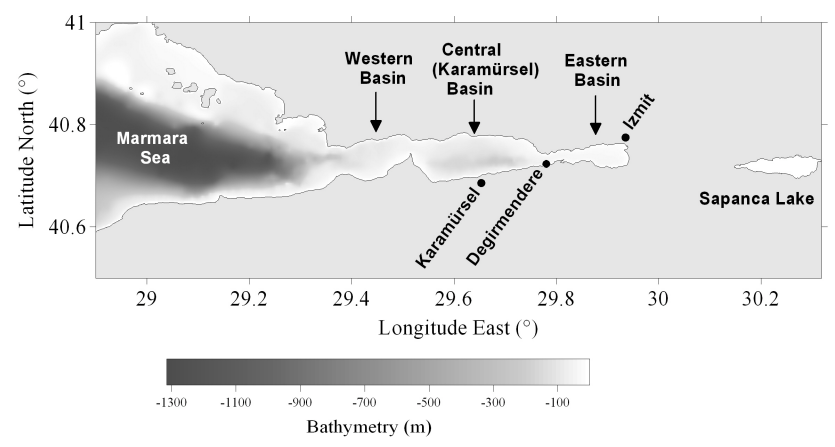

Fig. 5. The Izmit Gulf with its basins in the Maramara sea. Değirmendere is located in the south-eastern coast close to the end of the Karamürsel Basin.

where the unknown vectors $\mathbf{e}, \mathbf{X}, \mathbf{a}$ and $\mathbf{v}$ are on the 1.h.s. of the system, while vectors on the r.h.s. are known quantities. It is then easy to build a block matrix together with the corresponding block vectors in the form:

$$
\left(\begin{array}{ccccc}
\mathbf{\Gamma} & 0 & 0 & \mathbf{A}_{\mathbf{E}} & 0 \\
0 & \boldsymbol{\Gamma} & 0 & \mathbf{A}_{\mathbf{X}} & 0 \\
0 & -\mathbf{\Omega} \Delta x & \boldsymbol{\Gamma} & \mathbf{A}_{\mathbf{A}} & 0 \\
0 & \mathbf{I} & 0 & 0 & -\mathbf{F}_{\mathbf{1}}
\end{array}\right)\left(\begin{array}{c}
\mathbf{e} \\
\mathbf{X} \\
\mathbf{a} \\
\mathbf{p} \Delta x \\
\mathbf{v}
\end{array}\right)=\left(\begin{array}{c}
\mathbf{b}_{\mathbf{E}} \Delta x \\
\mathbf{b}_{\mathbf{X}} \Delta x \\
\mathbf{b}_{\mathbf{A}} \Delta x \\
\mathbf{F}_{\mathbf{2}} \mathbf{q}
\end{array}\right)
$$

which is a system of $4 N-1$ linear equations in $4 N-1$ unknowns and very suitable for inversion.

\subsection{Hypotheses on the unknown functions and related con- siderations}

The form (19) of the problem is quite flexible and allows one to easily explore different assumptions on the shape of the inter-slice force $X(x)$. In a first instance we assume a truncated three-term sine Fourier expansion for $X$ (i.e. we assume $m=3$ ), which is the same expression we already used in TM2006, where however we computed the solution through a less general ad-hoc method. More specifically we assume that

$X(x)=\sum_{k=1}^{3} \lambda_{k} \sin \left[k \pi \frac{x-x_{\text {beg }}}{x_{\text {end }}-x_{\text {beg }}}\right]$

and make the choice that $\lambda_{1}$ is a known parameter, while $\lambda_{2}$ and $\lambda_{3}$ are unknown quantities. Notice that the above position ensures that $X(x)$ vanishes at the end points of the domain as required by the condition (6). According to our notation we can write:

$$
\begin{gathered}
g_{i, 1}=\sin \left(\pi \frac{i}{N}\right) \quad i=1,2, . \cdots, N-1 \\
f_{i, 1}=\sin \left(2 \pi \frac{i}{N}\right) \quad i=1,2, . \cdots, N-1 \\
f_{i, 2}=\sin \left(3 \pi \frac{i}{N}\right) \quad i=1,2, . \cdots, N-1
\end{gathered}
$$


Table 2. Results for stratified soils obtained with all the methods used here. The three-layer stratification is shown in Fig. 4 . The homogeneous case is case 1 of Table $1\left(\gamma^{\prime}=25 \mathrm{kN} / \mathrm{m}^{3}, c^{\prime}=6 \mathrm{kPa}, \phi^{\prime}=25^{\circ}\right)$. Case 4 is heterogeneous in density $\left(\gamma_{1}^{\prime}=22 \mathrm{kN} / \mathrm{m}^{3}, \gamma_{2}^{\prime}=28 \mathrm{kN} / \mathrm{m}^{3}\right.$, $\left.\gamma_{3}^{\prime}=30 \mathrm{kN} / \mathrm{m}^{3}\right)$. Case 5 is heterogeneous in cohesion $\left(c_{1}^{\prime}=6 \mathrm{kPA}, c_{2}^{\prime}=100 \mathrm{kPa}, c_{3}^{\prime}=200 \mathrm{kPA}\right)$. Case 6 is heterogeneous as regards the friction angles $\left(\phi_{1}^{\prime}=10^{\circ}, \phi_{2}^{\prime}=15^{\circ}, \phi_{3}^{\prime}=35^{\circ}\right)$. Cells with the MLD values have bold characters.

\begin{tabular}{lllllllll}
\hline \multirow{2}{*}{ Method } & \multicolumn{2}{c}{ Case 1 } & \multicolumn{2}{c}{ Case 4 } & \multicolumn{2}{c}{ Case 5 } & \multicolumn{2}{c}{ Case 6 } \\
& F & $\delta$ & F & $\delta$ & F & $\delta$ & F & $\delta$ \\
\hline Spencer & 1.468 & 0.1052 & 1.523 & 0.1034 & 2.651 & 0.1164 & 2.012 & 0.1136 \\
T\&M & 1.409 & 0.0772 & 1.463 & 0.0760 & 2.588 & 0.0863 & 2.066 & 0.0771 \\
T\&M-new & 1.409 & 0.0776 & 1.463 & 0.0763 & 2.585 & 0.0864 & 2.060 & 0.0776 \\
T\&M-Xsin & 1.409 & 0.0776 & 1.463 & 0.0763 & 2.585 & 0.0864 & 2.060 & 0.0776 \\
T\&M-Xcos & $\mathbf{1 . 4 0 5}$ & $\mathbf{0 . 0 7 6 2}$ & $\mathbf{1 . 4 6 0}$ & $\mathbf{0 . 0 7 5 4}$ & $\mathbf{2 . 5 8 5}$ & $\mathbf{0 . 0 8 5 8}$ & $\mathbf{2 . 0 7 0}$ & $\mathbf{0 . 0 7 6 4}$ \\
T\&M-Ecos & 1.415 & 0.0836 & 1.471 & 0.0801 & 2.590 & 0.0895 & 2.100 & 0.0881 \\
T\&M-Acos & 1.412 & 0.0812 & 1.478 & 0.0803 & 2.600 & 0.0898 & 2.090 & 0.0823 \\
\hline
\end{tabular}

and identify $\lambda_{1}$ with $q=q_{1}$ and $\lambda_{2}$ and $\lambda_{3}$ with $v_{1}$ and $v_{2}$ respectively. Inversion of the system (19) provides a solution for any given choice of the known parameters, that are $F$ and $q_{1}$ in this case. In agreement with the adopted principle of MLD, the solving procedure consists (i) in solving the system (19) by letting these parameters to vary within reasonable intervals $I_{F}$ and $I_{q}$ that are obviously spanned at discrete steps, (ii) in computing the lithostatic deviation corresponding to each solution, i.e. in computing $\delta\left(F, q_{1}\right)$ within the 2-D space $I_{F} \times I_{q}$, which can be called the searching space, and eventually (iii) in finding the point in such a space where $\delta$ takes its minimum value. The corresponding value of $F$ is the searched value of the safety factor. It is worth stressing here once more the difference between the traditional methods of limit-equilibrium theory and ours. Those methods find only one solution of the equilibrium equations and take the corresponding value of $F$ as the safety factor of the slope. But we know that a solution can be found for any point of the searching space. Therefore, since the interval $I_{F}$ may be shown to include the discriminant value of unity, the consequence is that one has no means to judge on the stability of a slope, unless one invokes an additional criterion, such as the MLD principle.

In Table 1 we show the results of our computations applied to the body sketched in Fig. 2, which is the same body the reader can find in TM2006. These results, that will be designated by T\&M-new, are compared with the one that were obtained in TM2006 and that are here denoted by TM. As expected, they practically coincide and the slight differences are uniquely due to small numerical rounding errors associated with the fact that in TM2006 we solve the same basic set of equations by using an ad-hoc semi-analytical method, while here we invert system (19) by a standard numerical real-matrix inversion routine.

Since, given a series expansion, one can choose freely the two terms of the series whose coefficients are unknown, we explore the effect of a different choice. In the following we still make recourse to the three-term sine Fourier expansion, but we take:

$g_{i, 1}=\sin \left(3 \pi \frac{i}{N}\right) \quad i=1,2, \ldots, N-1$

and

$f_{i, 1}=\sin \left(\pi \frac{i}{N}\right) \quad i=1,2, \ldots, N-1$

$f_{i, 2}=\sin \left(2 \pi \frac{i}{N}\right) \quad i=1,2, . \cdots, N-1$

The corresponding solutions will be denoted by T\&M-Xsin in this paper. A further explored hypothesis is to consider a different set of base functions $g$ and $f$. Instead of sine functions, we can take cosine functions multiplied by the local normalised weight to ensure fulfilment of the boundary condition (6), i.e. we assume:

$g_{i, 1}=\frac{w\left(x_{i}\right)}{w_{\max }} \cos 2 \pi \frac{i}{N} \quad i=1,2, . \cdots, N-1$

and

$\begin{aligned} f_{i, 1} & =\frac{w\left(x_{i}\right)}{w_{\max }} \quad i=1,2, \ldots, N-1 \\ f_{i, 2} & =\frac{w\left(x_{i}\right)}{w_{\max }} \cos \pi\left(\frac{i}{N}\right) \quad i=1,2, \ldots, N-1\end{aligned}$

Here $w_{\max }$ is defined as the $\max \left\{w\left(x_{i}\right)\right\} i=1,2, \ldots, \mathrm{N}-1$. The related solutions will be designated by T\&M-Xcos.

All the above hypotheses involve assumptions on the interslice vertical forces $X(x)$ and require the inversion of the system of Eq. (19). Our formulation enables one to make hypotheses concerning also the other unknown functions $E(x)$ and $A(x)$. And it is very easy to see that the corresponding 
system of equations can be written in the following "block" forms:

$$
\begin{aligned}
& \left(\begin{array}{ccccc}
\boldsymbol{\Gamma} & 0 & 0 & \mathbf{A}_{\mathbf{E}} & 0 \\
0 & \boldsymbol{\Gamma} & 0 & \mathbf{A}_{\mathbf{X}} & 0 \\
0 & -\boldsymbol{\Omega} \Delta x & \boldsymbol{\Gamma} & \mathbf{A}_{\mathbf{A}} & 0 \\
\mathbf{I} & 0 & 0 & 0 & -\mathbf{F}_{\mathbf{1}}
\end{array}\right)\left(\begin{array}{c}
\mathbf{e} \\
\mathbf{X} \\
\mathbf{a} \\
\mathbf{p} \Delta x \\
\mathbf{v}
\end{array}\right)=\left(\begin{array}{c}
\mathbf{b}_{\mathbf{E}} \Delta x \\
\mathbf{b}_{\mathbf{X}} \Delta x \\
\mathbf{b}_{\mathbf{A}} \Delta x \\
\mathbf{F}_{\mathbf{2}} \mathbf{q}
\end{array}\right) \\
& \left(\begin{array}{ccccc}
\boldsymbol{\Gamma} & 0 & 0 & \mathbf{A}_{\mathbf{E}} & 0 \\
0 & \boldsymbol{\Gamma} & 0 & \mathbf{A}_{\mathbf{X}} & 0 \\
0 & -\boldsymbol{\Omega} \Delta x & \boldsymbol{\Gamma} & \mathbf{A}_{\mathbf{A}} & 0 \\
0 & 0 & 0 & \mathbf{I} & -\mathbf{F}_{\mathbf{1}}
\end{array}\right)\left(\begin{array}{c}
\mathbf{e} \\
\mathbf{X} \\
\mathbf{a} \\
\mathbf{p} \Delta x \\
\mathbf{v}
\end{array}\right)=\left(\begin{array}{c}
\mathbf{b}_{\mathbf{E}} \Delta x \\
\mathbf{b}_{\mathbf{X}} \Delta x \\
\mathbf{b}_{\mathbf{A}} \Delta x \\
\mathbf{F}_{\mathbf{2}} \mathbf{q}
\end{array}\right)
\end{aligned}
$$

We have made experiments of both types. In particular, we have selected the three-term expansion given by the cosine functions (20)-(22) and inverted the system (23) when the position regarded the horizontal forces $E(x)$, while we have inverted the system (24) when the position regarded the torque $A(x)$. The results will be referred as T\&M-Ecos in the first case and as T\&M-Acos in the second.

The formulation of the limit-equilibrium problem proposed here leads to the inversion of the "block" system of equations in one of the three forms (19), (23) and (24). We stress that this is a relevant improvement on previous methods: not only on the traditional methods, but also on the TM2006 formulation, since the present version combines the advantage of being computationally fast (as most of the other methods) with a great flexibility, since it allows one to explore quite easily different assumptions on the shape of the unknown functions.

It is relevant also to point out that each hypothesis leads to a different solution for the safety factor $F$, since this is obtained by minimising the lithostatic deviation $\delta$ within the searching space. In the general case of an $m$-term expansion like the position (16) the searching space will have dimension $m-1$, since the involved parameters are the $m-2$ vector $\boldsymbol{q}$ and $F$. The fact that we have a multiplicity of results for $F$ is not crucial since we may resolve such an apparent ambiguity by making recourse once more to the MLD principle. Indeed we will select as the best solution for $F$, the one that is associated with lowest value of $\delta$. This means that there is no way to judge the goodness of a hypothesis of type (16) a priori. Each of these can be seen as a way to explore a portion of the searching space, and a posteriori we can consider that the best assumption is the one providing the minimum $\delta$. Of course, according to this point of view, there is no certainty that the minimum value for $\delta$ we have found by exploring a given set of hypotheses (one or more), is the absolute minimum, i.e. there is no certainty that other unexplored hypotheses could provide smaller lithostatic deviations and correspondingly different solutions for the safety factor. This issue is inherent to many minimisation problems and is in principle unavoidable for the limit-equilibrium theory. This observation casts a better light to the limitations of the traditional limit-equilibrium methods that compute only

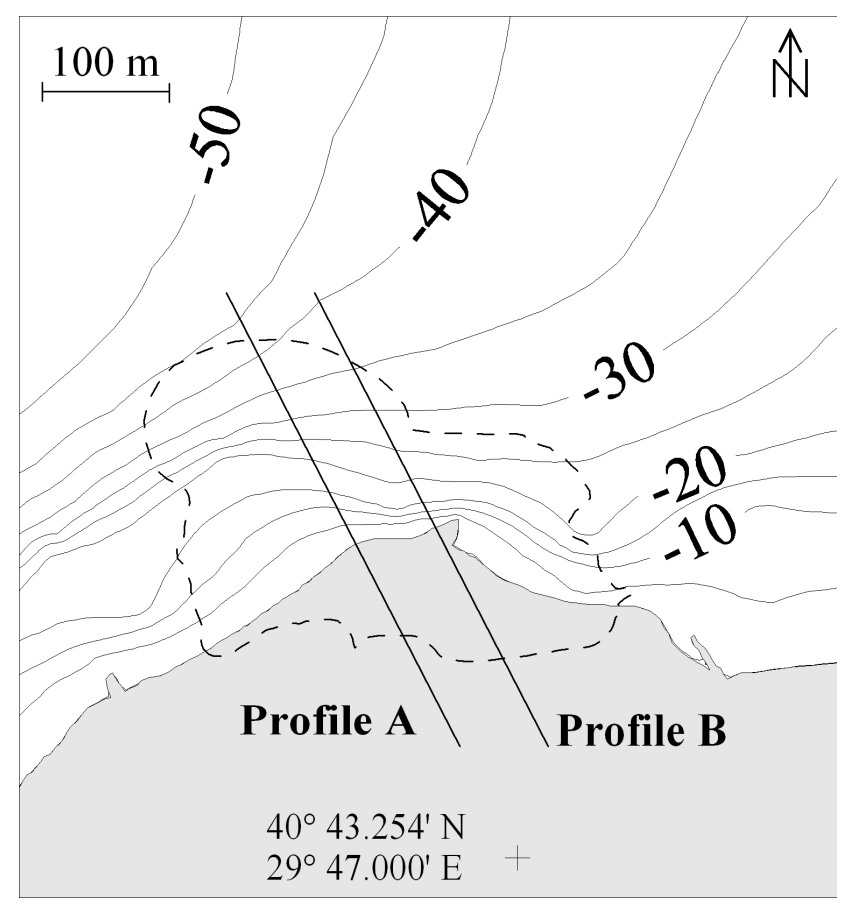

Fig. 6. Değirmendere coastline before the slide (solid line) and footprint of the sliding mass body (dashed line) as reconstructed by Rathje et al. (2004) and by Cetin at al. (2004). Profiles A and B correspond to the vertical-cross sections analysed in the paper.

one solution for the problem, which can be rephrased by stating that they restrict their searching space to only one point, which is a not advisable practice to find a point of minimum.

\section{Applications to idealized cases}

The cases taken into account for the application of our method are initially the same as those that were analysed in TM2006, since this enables us to make proper comparisons. A slope of about $30^{\circ}$ with an arc-like sliding surface is represented in Fig. 2. The body is homogeneous and may be partially submerged under a layer of water with a possible piezometric level that is depicted by a dashed piecewise line. This profile is studied for three different situations: case 1 corresponds to a dry body with null pore pressure and with no external forces applied; case 2 is the case of a body under the load of a thin water layer applied on the toe side; in case 3 a seismic load is considered $\left(k=0.368, \psi=42.8^{\circ}\right)$. The stability is studied by means of the classical method by Spencer and by means of the TM method (Tinti and Manucci, 2006), and, in addition, by using the five more different approaches illustrated in the previous section, i.e. by inverting the "block" system. The discretization of the computational domain $\left[x_{\text {beg }}, x_{\text {end }}\right]$ is made by using a grid of $N+1=51$ nodes in all the following examples. 
Table 3. Stability results for the Degirmendere slide body in the pre-earthquake conditions. Profiles A and B are shown in Figs. 6 and 7. All methods give equivalent results: the body is extremely stable on both profiles. Cells with bold characters contain the MLD values.

\begin{tabular}{lllll}
\hline \multirow{2}{*}{ Method } & \multicolumn{2}{c}{ Profile A } & \multicolumn{2}{c}{ Profile B } \\
& F & $\Delta$ & F & $\delta$ \\
\hline Spencer & 5.963 & 0.0680 & 7.127 & 0.0525 \\
T\&M & $\mathbf{5 . 9 6 6}$ & $\mathbf{0 . 0 6 7 2}$ & $\mathbf{7 . 1 1 5}$ & $\mathbf{0 . 0 5 2 3}$ \\
T\&M-new & $\mathbf{5 . 9 6 1}$ & $\mathbf{0 . 0 6 7 2}$ & $\mathbf{7 . 1 2 7}$ & $\mathbf{0 . 0 5 2 3}$ \\
T\&M-Xsin & $\mathbf{5 . 9 6 1}$ & $\mathbf{0 . 0 6 7 2}$ & $\mathbf{7 . 1 2 7}$ & $\mathbf{0 . 0 5 2 3}$ \\
T\&M-Xcos & 5.961 & 0.0672 & 7.127 & 0.0523 \\
T\&M-Ecos & 5.956 & 0.0976 & 7.125 & 0.1938 \\
T\&M-Acos & 5.939 & 0.3049 & 7.126 & 0.7750 \\
\hline
\end{tabular}

All results are summarized in Table 1 where the values of the minimum lithostatic deviations and of the corresponding safety factors are given. Technically, the complete solution includes the further specification of the computed unknown functions $E(x), X(x), \mathrm{A}(x)$ and $P(x)$. For case 1, these curves are provided in Fig. 3. The body results to be stable in case 1 and even more stable in case 2, where the water load stabilizes the slope, while it is unstable in case 3 due to the seismic load.

As it may be seen from Table 1, the classical Spencer's method gives results quite different from all our approaches, both in terms of MLD (remarkably higher) and in terms of $F$ (higher). Judged through the MLD principle, this method results to be the worst. On the other hand, the results of all our methods are quite close to one another. As already remarked, the methods T\&M and T\&M-new compute the solution exactly to the same problem, but via different numerical algorithms. Hence, the differences in the corresponding results are only due to numerical rounding errors. It is further interesting to note that the methods T\&M-new and T\&M-Xsin assume the same three-term expansion for $X(x)$, though using different choices for known and unknown coefficients. The results are almost identical, which is explained by the fact that these methods explore the same searching space to find the MLD, though by means of a different searching grid. Further, the worst results for our methods are the ones deriving from the weighted cosine expansions of the function $E$ and $A$ (T\&M-Ecos, T\&M-Acos). Finally, we observe that the minimum values of $\delta$ are obtained by the method T\&M$\mathrm{X} \cos$ for all three cases, which is suggestive that the weighted cosine expansion of $X$ is the best possible assumption among the ones examined here.

Looking at the graphs of Fig. 3, it is clear that the three sinusoidal expansion methods (T\&M, T\&M-new, T\&M-Xsin) are almost equivalent. The weighted cosine expansion for the force $X$, which is our best solution, departs slightly from the others in the up-hill part of the slide regards the force
Table 4. Stability results for the Değirmendere slide body under conditions presumably acting during the earthquake $(k=0.45, \psi=-$ $22^{\circ}$ ) and the consequent tsunami produced by the earthquake itself (sea level lowers by $1 \mathrm{~m}$ ). All methods, including the traditional Spencer's method, produce similar values for the factor of safety, and the conclusion is that both profiles are unstable. Cells with bold characters contain the MLD values.

\begin{tabular}{lllll}
\hline \multirow{2}{*}{ Method } & \multicolumn{2}{c}{ Profile A } & \multicolumn{2}{c}{ Profile B } \\
& F & $\delta$ & F & $\delta$ \\
\hline Spencer & 0.908 & 0.1728 & 0.989 & 0.1714 \\
T\&M & $\mathbf{0 . 9 0 6}$ & $\mathbf{0 . 1 6 2 1}$ & 0.989 & 0.1630 \\
T\&M-new & 0.906 & 0.1623 & $\mathbf{0 . 9 8 8}$ & $\mathbf{0 . 1 6 2 5}$ \\
T\&M-Xsin & 0.906 & 0.1623 & 0.988 & 0.1625 \\
T\&M-Xcos & 0.906 & 0.1623 & 0.988 & 0.1626 \\
T\&M-Ecos & 0.906 & 0.1631 & 0.988 & 0.1696 \\
T\&M-Acos & 0.904 & 0.1861 & 0.987 & 0.2592 \\
\hline
\end{tabular}

and torque functions, but it is quite similar as far as the bottom pressure $P$ is concerned. Worth of notice is that for all the cosine methods (T\&M-Xcos, T\&M-Ecos, T\&M-Acos) there is an irregularity of the curves in correspondence with the thinning of the sliding mass at the horizontal distance of about $75 \mathrm{~m}$ (see Fig. 2). This is due to the fact that, in this expansion, cosines are corrected by a normalised weight (proportional to the slide thickness), that changes abruptly around that value of the abscissa. We remark further that the last two methods (T\&M-Ecos, T\&M-Acos) give curves very different from all the others, with the largest discrepancies observable for the torque profiles.

\subsection{Stratification}

The stratification of the soil is incorporated in the formulation of the limit-equilibrium problem presented here through the coefficients $\alpha(x)$ and $\beta(x)$ of the formulas (9) and hence entered in the final discrete system of Eqs. (19), (23) and (24) through the matrices $\mathbf{A}_{\mathbf{E}}, \mathbf{A}_{\mathbf{X}}$, and $\mathbf{A}_{\mathbf{A}}$ and the vectors $\mathbf{b}_{\mathbf{E}}, \mathbf{b}_{\mathbf{X}}$, and $\mathbf{b}_{\mathbf{A}}$. Therefore, system (19), (23) and (24) is perfectly suitable to handle also the stability of layered slopes, which is often of great interest. We give some examples of application by using the stratified body portrayed in Fig. 4 . We examine three cases of stratification we call cases 4, 5 and 6 . Taking the previous case 1 (homogeneous dry body with no external forces applied) as reference, for each case we vary only one parameter: in case 4 the density is taken to increase with depth, in case 5 the cohesion increases with depth and in case 6 the angle of friction. They all are compared with the homogeneous case. In case 4 the sliding mass is lighter than for the reference homogeneous case, and consequently the slope is slightly more stable. In case 5 , the cohesion is remarkably higher, and hence the mass is by far more stable. In case 6 the larger angles of friction are again an element 

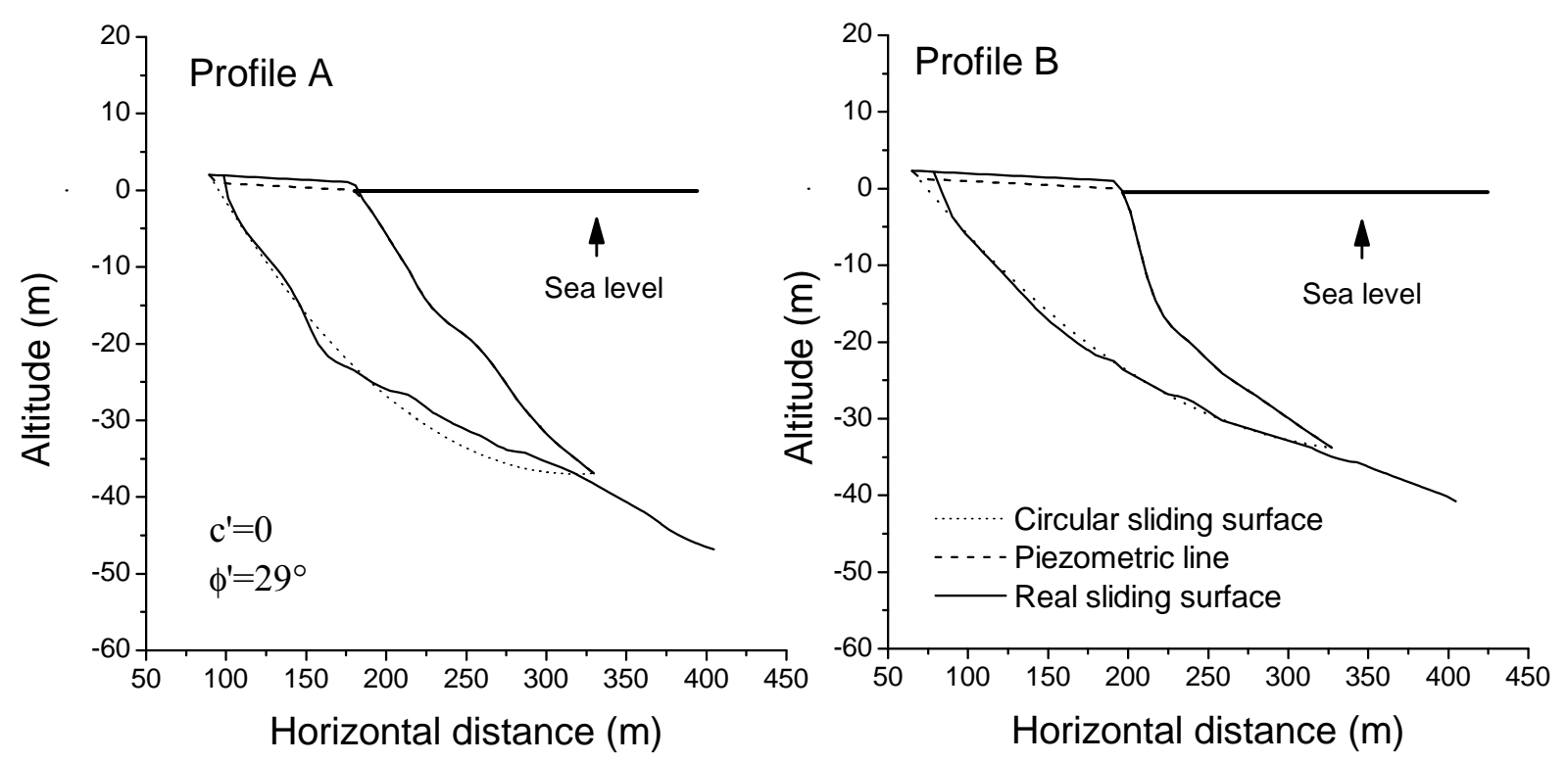

Fig. 7. Vertical cross sections of the profiles A and B of Fig. 6. The soil parameter c', $\phi$ ', $\rho$ are constant over the sliding mass, with respective values of $0 \mathrm{kPa},-22^{\circ}$ and $20 \mathrm{kNm}^{-3}$. The sliding surfaces are assumed to be circular (dashed line) and the sliding bodies are partly submerged.

of stabilisation for the body. All results are given in Table 2 . Very interestingly we confirm here that Spencer's method departs from all the others, that all the sine expansion cases give similar results, and that the best results are obtained through the weighted cosine expansion of the vertical inter-slice force $X(\mathrm{~T} \& \mathrm{M}-\mathrm{X} \cos )$.

\subsection{A real case}

In this section we apply the block-matrix method to a real case, that is the case of a slide that was released in Değirmendere, a coastal village in the Izmit Gulf, Turkey, as the result of a disastrous $M=7.4$ earthquake, that affected the north-western part of Turkey on 17 August 1999. Değirmendere is located in the south coast of the Izmit Gulf between the Karamürsel Basin and the Eastern Basin (see Fig. 5). The slide involved a segment of coast about $300 \mathrm{~m}$ long and $75 \mathrm{~m}$ wide, and carried into the sea a multi-storey hotel and two adjacent buildings. It produced a local tsunami. In Fig. 6 the coastline before and after the slide is sketched, together with two profiles A and B intersecting the coast, along which we take the vertical cross-sections depicted in Fig. 7 (Tinti et al., 2006). The earthquake produced a tsunami that was observed in the entire Izmit bay. The tsunami was not catastrophic, with measured run-up heights comprised in the range of $1-3 \mathrm{~m}$. The stability analysis that was conducted by Tinti et al. (2006) showed that the earthquake shaking, with peak ground acceleration estimated to be about $0.45 \mathrm{~g}$, was the cause of the slide, and in turn of the associated tsunami that added its effect locally (run-up heights larger than $10 \mathrm{~m}$ ) to the one produced by the earthquake (see also Wrigth and Rathje, 2003). It was further speculated that the lowering of the sea level associated with the earthquake tsunami could have acted as an additional factor of destabilisation for the slide.

The two cross-sections A and B have been analysed with the new computational approach described before and the results are synthesised in Tables 3 and 4. Two cases are considered for both profiles: first, the stability of the slope is evaluated in the pre-earthquake condition with no seismic shaking and no effect of the seismic-origin tsunami (Table 3); secondly, the stability is analysed under the condition of an active seismic load $\left(k=0.45, \psi=-22^{\circ}\right)$ and of an ongoing tsunami causing a destabilising sea level decrease of $1 \mathrm{~m}$ (Table 4). The material properties of the body were measured by Cetin et al. (2004) during a post-earthquake survey. Comparing the results of all methods, one sees that the methods providing the highest values of the lithostatic deviation are confirmed to be Spencer, T\&M-Ecos and T\&M-Acos. However, one also finds that the computed values of the factor of safety are quite similar, though the values of the $\delta$ depart somewhat from one another. This means that for this particular application using one method or another is nearly equivalent for practical purposes.

\section{Conclusions}

The study of stability of a slope is an issue of great relevance. The limit-equilibrium method posed the basis to make stability analysis, but the classical methods show drawbacks mostly related with the non-uniqueness of the solution. The 
MLD principle provides a criterion to find the best solution and the corresponding safety factor for the slope under study. In this paper we have worked within the frame of the MLD approach and on discretising the basic system of equilibrium equations. We have obtained a linear set of algebraic equations in the block matrix form (19), (23) and (24) that is quite easy to solve by the standard real-matrix inversion codes. One of the most relevant result of our analysis is the expression of the problem through the formulation (19), (23) and (24). It has a number of great advantages: 1) it may be applied to a wide range of slopes and of conditions, since it accounts for dry and wet soils, for external distributed loads such as those exerted by a water layer or by seismic shaking; 2) it handles bodies with arbitrary geometry, which includes arbitrary bottom profiles; 3 ) it accounts also for an arbitrary body stratification (which means that, since layers can be separated by arbitrary interfaces and be made as thin as we wish, it can also be adapted to treat a totally heterogeneous body with variables depending on the horizontal and vertical coordinates $x$ and $z$ ); 4) the formulation is flexible enough to allow one to make a wide range of assumptions on the unknown vectors by expanding one of these (either $X$, or $E$, or $A$ ) over a set of $m$ base-functions, and consequently to explore the corresponding $(m-1) \mathrm{D}$ searching space. For illustrative reasons we restricted all our applications to $m=3$ expansions which lead to 2-D searching space of the type $I_{F} \times I_{q}$.

Comparison of our results with traditional methods is limited to the Spencer's method that was seen to be one of the best classical approaches (TM2006). This comparison shows that Spencer's method provides always larger values of lithostatic deviation and hence, if the MLD principle is adopted, worse determinations of the safety factor. For all cases examined in this paper, expansions of the unknowns $E$ and $A$ seem to miss the MLD and then are probably not advisable. When we considered the homogeneous body already studied in TM2006, we found that the most convenient assumption is the one we called $\mathrm{T} \& \mathrm{M}-\mathrm{X} \cos$, and the same conclusion was also reached for the ideal stratified bodies (heterogeneous as regards either density, or cohesion, or friction angle). When we considered the real case of the Değirmendere slide that was caused by a tsunamigenic earthquake and itself caused a local tsunami, we found that one can confirm the same ranking of the methods in terms of MLD, but, in spite of this, all methods, inclusive Spencer's, lead to very similar values of the safety factor. The fact that $F$ is rather insensitive to $\delta$, can be also stated in the inverse way that very small variations of $F$ produce very large changes of $\delta$. Whether this is due to the geometrical or physical properties of the body or is the apparent effect of the limitedness of the searching space spanned in this application is a question that can be answered by making further assumptions on the unknowns, e.g. by increasing the number $m$ of the coefficients in the proposed expansions, which is a subject of further research.
Acknowledgements. This research has been financed on funds from the European project TRANSFER n.037058 and the project FIRB RBAP04EF3A_004 of the Italian Ministry of University and Research (MIUR).

Edited by: K.-T. Chang

Reviewed by: A. Volkwein and another anonymous referee

\section{References}

Bishop, A. W.: The use of the slip circle in the stability analysis of slopes, Geotechnique, 5, 7-17,1955

Cetin, K. O., Isik, N., Unutmaz, B.: Seismically induced landslide at Deirmendere Nose, Izmit bay during Kocaeli (Ýzmit)Turkey earthquake, Soil Dynamics and Earthquake Engineering, 24, 189-197, 2004.

Chen, Z. and Morgenstern, N. R.: Extension to the generalized method of slices for stability analysis, Can. Geotech. J., 20, 104$119,1983$.

Chen, Z., Mi, H., and Wang, X.: A three-dimensional limit equilibrium method for slope stability analysis, Chinese J. Geotech.Engrg., 23, 525-529, 2001.

Duncan, J. M. and Wright, S. G.: The accuracy of equilibrium methods of slope stability analysis, Engrg. Geol., 16, 5-17, 1980.

Fellenius, W.: Erdstatische Berechnungen mit Reibung und Kohasion, Ernst, Berlin (in German), 1927.

Graham, J.: Methods of stability analysis, In "Slope instability", edited by D. Brunsden and D.B. Prior, Wiley-Intersciences Publication, Wiley \& Sons, Chichester, 171-215, 1984.

Janbu, N.: Slope stability computations, Soil Mech. And Found. Engrg. Rep., The Technical University of Norway, Trondheim, Norway, 1968.

Jiang, J. C. and Yamagami, T.: Three-dimensional slope stability analysis using an extended Spencer method, Soils and Foundations, 44, 127-135, 2004.

Karaulov, A. M.: Statement and solution of the stability problem for slopes and embankments as a linear-programming problem, Soil Mechanics and Foundation Engineering, 42, 75-80, 2005.

Leshchinsky, D. and Huang, C.: Generalized three dimensional slope stability analysis, J. Geotech. Engrg., ASCE, 118, 17481764, 1992.

Morgenstern, N. R. and Price, V. E.: The analysis of the stability of general slip surfaces, Geotechnique, 15, 79-93, 1965.

Pink, M. N.: Analysis of slope stability by the method of limiting equilibrium, Soil Mechanics and Foundation Engineering, 44, 99-104, 2007.

Rathje, E. M., Karatas, I., Wright, S. G., and Bachhuber, J.: Coastal Failures during the 1999 Kocaeli Earthquake in Turkey, Soil Dyn. Earthqu. Eng., 24(9-10), 699-712, 2004.

Spencer, E.: A method of analysis of the stability of embankments assuming parallel interslice forces, Geotechnique, 17, 11-26, 1967.

Tinti, S. and Manucci, A.: Gravitational stability computed through the limit equilibrium method revisited, Geophysical International Journal, 164, 1-14, 2006.

Tinti, S., Armigliato, A., Manucci, A., Pagnoni, G., Zaniboni, F., Yalçiner, A. C., and Altinok, Y.: The generating mechanisms of the August 17, 1999 Izmit bay (Turkey) tsunami: regional 
(tectonic) and local (mass instabilities) causes, Marine Geology, 225, 311-330, 2006.

Wright, S. G. and Rathje, E. M.: Triggering mechanisms of slope instability and their relationship to earthquakes and tsunamis, Pure and Applied Geophysics, 160, 1865-1877, 2003.

Zheng, H., Liu, D. F., and Li, C. G.: On the assessment of failure in slope stability analysis by finite element method, Technical note, Rock Mechanics and Rock Engineering, doi:10.1007/s00603007-0129-8, 2007.
Zhu, D. Y., Lee, C. F., and Jiang, H. D.: Generalised framework of limit equilibrium methods for slope stability analysis, Geotechnique, 53, 377-395, 2003. 